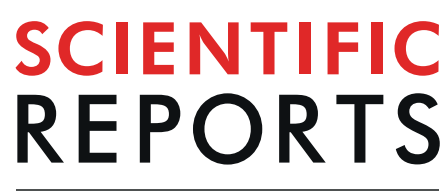

natureresearch

\title{
Diat.barcode, an open-access curated barcode library for diatoms
}

\author{
Frédéric Rimet $\mathbb{B}^{1,2^{*}}$, Evgenuy Gusev ${ }^{3}$, Maria Kahlert ${ }^{4}$, Martyn G. Kelly ${ }^{5}$, Maxim Kulikovskiy ${ }^{3}$, \\ Yevhen Maltsev $\mathbb{D}^{3}$, David G. Mann $\mathbb{1}^{6,7}$, Martin Pfannkuchen $\mathbb{1 0}^{8}$, Rosa Trobajo ${ }^{7}$, \\ Valentin Vasselon ${ }^{1,2}$, Jonas Zimmermann ${ }^{9}$ \& Agnès Bouchez ${ }^{1,2}$
}

Diatoms (Bacillariophyta) are ubiquitous microalgae which produce a siliceous exoskeleton and which make a major contribution to the productivity of oceans and freshwaters. They display a huge diversity, which makes them excellent ecological indicators of aquatic ecosystems. Usually, diatoms are identified using characteristics of their exoskeleton morphology. DNA-barcoding is an alternative to this and the use of High-Throughput-Sequencing enables the rapid analysis of many environmental samples at a lower cost than analyses under microscope. However, to identify environmental sequences correctly, an expertly curated reference library is needed. Several curated libraries for protists exists; none, however are dedicated to diatoms. Diat.barcode is an open-access library dedicated to diatoms which has been maintained since 2012. Data come from two sources (1) the NCBI nucleotide database and (2) unpublished sequencing data of culture collections. Since 2017, several experts have collaborated to curate this library for $r b c L$, a chloroplast marker suitable for species-level identification of diatoms. For the latest version of the database (version 7), 605 of the 3482 taxonomical names originally assigned by the authors of the $r b c L$ sequences were modified after curation. The database is accessible at https:// www6.inra.fr/carrtel-collection_eng/Barcoding-database.

The Bacillariophyta (diatoms) is a particularly species diverse phylum of microalgae with an estimated 100,000 species ${ }^{1}$. This algal clade is present in terrestrial, freshwater and marine habitats and each taxon occupies a particular niche ${ }^{2}$. The advantage of these properties of ubiquity and taxonomic diversity were noticed a long time ago. Indeed, the first studies demonstrating the effect of pollution on freshwater diatom communities were over a century ago $^{3}$ and several methods for using diatoms to assess pollution were proposed in the second half of the twentieth century $\left(\right.$ e.g. $\left.{ }^{4-6}\right)$. Such tools have been used routinely worldwide ${ }^{7}$ in particular to fulfill national or transnational directives (e.g. the Water Framework Directive in Europe ${ }^{8}$ and the National Water-Quality Assessment Program in the USA ${ }^{9}$ ). Moreover, diatoms often represent an important part of the total biomass in aquatic ecosystems and make a major contribution to global productivity, and therefore cannot be ignored in ecological studies (e.g. ${ }^{10}$ ).

Classically, diatoms are identified by looking at the gestalt and morphology of their siliceous exoskeleton (frustule) using a microscope. Standard procedure using diatoms as ecological indicators require counting and determining several hundreds of frustules under a microscope ${ }^{11}$. Such procedures are time consuming and, when several thousands of sites need to be monitored, the time and cost of such approaches are substantial (e.g. ${ }^{12,13}$ ). Moreover, because of the difficulties involved in differentiating between some diatom species, there can be considerable variation between assessment results, even when experienced analysts are involved (e.g. ${ }^{14,15}$ ).

DNA-barcoding is a taxonomic method that uses a short stretch of DNA to identify species ${ }^{16}$ through comparison with a curated library of reference sequences (i.e. DNA barcodes). Early tests of DNA barcoding to identify animals ${ }^{16}$ and plants ${ }^{17}$ showed a promising ability for non-taxonomists to identify organisms. It was then applied to diatoms ${ }^{18,19}$ and several DNA-markers were evaluated (18S and 28S rDNA, cox1, ITS rDNA, rbcL). $18 \mathrm{~S}$ V4 and

${ }^{1}$ INRA, UMR CARRTEL, 75bis av. de Corzent - CS 50511, FR-74203, Thonon les Bains cedex, France. ${ }^{2}$ University of Savoie Mont-Blanc, UMR CARRTEL, FR-73370, Le Bourget du Lac, France. ${ }^{3}$ Institute of Plant Physiology, Russian Academy of Sciences, 127276, Moscow, Russia. ${ }^{4}$ Swedish University of Agricultural Sciences, Department of Aquatic Sciences and Assessment, PO Box 7050, SE- 750 07, Uppsala, Sweden. ${ }^{5}$ Bowburn Consultancy, 11 Monteigne Drive, Bowburn, Durham, DH6 5OB, UK. ${ }^{6}$ Royal Botanic Garden Edinburgh, Edinburgh, EH3 5LR, Scotland, UK. ${ }^{7}$ Marine and Continental Waters, Institute for Food and Agricultural Research and Technology (IRTA), Crta de Poble Nou Km 5.5, Sant Carles de la Ràpita, Catalonia, Spain. ${ }^{8}$ Institut Ruđer Bošković, Giordano Paliaga 5, 52210, Rovinj, Croatia. ${ }^{9}$ Botanischer Garten und Botanisches Museum Berlin-Dahlem, Freie Universität Berlin, Königin-Luise-Str. 6-8, 14195, Berlin, Germany. *email: frederic.rimet@inra.fr 
$r b c \mathrm{~L}$ are the most frequently used markers at present (e.g. $\left.{ }^{20,21}\right)$. When affordable High-Throughput-Sequencing (HTS) methods arrived in 2005, the possibility of using barcoding to analyse environmental samples composed by a mixture of taxa became real. This method, called metabarcoding ${ }^{22}$ uses HTS to sequence environmental samples in order to obtain a large quantity of sequences per run. By comparing each HTS sequence to the barcode reference library, the composition of the environmental community can be ascertained. For diatoms this method was first tested on mock communities made of already-barcoded strains ${ }^{12}$ and then on natural communities from several temperate or tropical rivers ${ }^{23,24}$, lakes ${ }^{25,26}$, marine habitats ${ }^{27,28}$ and even ancient diatom DNA (e.g. ${ }^{29,30}$ ). When diatoms are the sole focus of the study, a short region with high discrimination capacity is needed and partial $r b c \mathrm{~L}$ (chloroplastic marker) has proven to be suitable (e.g. ${ }^{12,31}$ ); this is sequenced using specific primers for diatoms. In some other studies the V4 region of $18 \mathrm{~S}$ (ribosomal marker) has been sequenced; however, its discrimination capacity is slightly lower than that of $r b c \mathrm{~L}^{12}$. On the other hand, the use of generic primers for this region enables a larger range of diversity to be covered than solely diatoms.

The general point raised by all these metabarcoding studies is that an Achilles heel of metabarcoding is the barcode reference library. It must be as comprehensive as possible in order to be able to assign a high proportion of environmental sequences to known taxa, and it requires regular expert curation in order to maintain its quality (i.e. taxonomic assignments, sequences quality and traceability of data and metadata). Several databases curated by experts already exist. Some are general DNA barcoding libraries of protists diversity such as SILVA ${ }^{32}$ and PR2 ${ }^{33}$. Others are more focused, e.g. PhytoREF for photosynthetic organisms ${ }^{34}$, PFR2 for planktonic foraminifera (Morard et al..$^{35}$ ), EukRef-Ciliophora for ciliates ${ }^{36}$ and Dinoref for Dinophyceae ${ }^{37}$, although the last database has now been integrated in PR2.

Diat.barcode is a reference library dedicated to diatoms with fine-tuned taxonomy and curation at genus and species level, which is maintained since 2012. This paper describes this open-access barcode reference library and its curation workflow. This library has been used in several ecological studies for rivers and lakes using diatoms (e.g. ${ }^{12,23,25,26,31,38,39}$ ). Diat.barcode gathers data and metadata for the $r b c \mathrm{~L}$ marker and also, to a lesser extent, for $18 \mathrm{~S}$, $28 \mathrm{~S}$, cox1 and ITS (only for a few cultures). It is freely accessible through https://www6.inra.fr/carrtel-collection eng/Barcoding-database. An earlier paper has described the former versions of this database (v1 to v6), it was called R-Syst:: diatom ${ }^{40}$. This new paper describes the evolution of the curation procedure, which has been simplified and which is now done collectively by several European experts in diatom taxonomy and phylogeny. Moreover, the name of the database was changed from R-Syst::diatom to Diat.barcode since it moved from a French initiative to an international collaboration. This collective curation ensures a more robust outcome and employs a procedure very similar to that used in EukRef, a community of people with expertise in diverse eukaryotic lineages to curate $18 \mathrm{~S}$ rDNA data and using phylogenetic methods with the goal of creating a curated reference library of eukaryotes ${ }^{41}$.

Our aim was to produce a curated reference library for diatom metabarcoding. Here we present barcode sources, metadata associated with the barcodes, data curation procedures, and information on data storage and accessibility. Then we show the results of the latest curation of the reference library (version 7) as well as its contents. A description of the release of a ready-to-use database for metabarcoding is also given, namely Rsyst::diatom_rbcl_align_312bp, which is an aligned subset of Diat.barcode.

\section{Results and Discussion}

Example of the curation of diat.barcode version 7 (February 2018). The curation of Diat.barcode version 7 was carried out at the start of 2018. Seven different experts worked on it, each focusing on clades corresponding to their specialist knowledge. For instance R. Trobajo and D. Mann curated the clade of the Nitzschia s.l. because they have published several papers on this genus (e.g. $\left.{ }^{42-45}\right)$, J. Zimmermann curated the monoraphids because his team published several papers on this group (e.g. $\left.{ }^{24,46}\right)$, M. Pfannkuchen curated newly-described marine diatom sequences, reflecting his laboratory's interests (e.g. ${ }^{47-49}$ ) and M. Kahlert the Fragilaria genus ${ }^{50}$.

During this process, 703 sequences were curated according to the procedures described above and summarized in Table 1. Among these newly-retrieved sequences, five were removed because the sequence name was too different from the phylogenetic neighbors and no original material was available to check. On the other hand, 698 sequences were kept. Globally, in Diat.barcode version 7, for $r b c L$ gene, 605 of the 3482 original names given by the authors of the sequence were modified (i.e. 17\%).

Content of the database. Number of sequences and geographical location of the sampling sites. The number of sequences publicly available for $r b c \mathrm{~L}$ is given in Table 2. Sequences from the TCC, the Laboratory of Molecular Systematics of Aquatic plants, Institute of Plant Physiology, Russian Academy of Sciences (curated by Maxim Kulikovsky) and the UK barcoding project (funded by the UK Environment Agency) represent almost $30 \%$ of the total. Only $18.7 \%$ (210) of the sequences of the TCC and UK barcoding project have so far been deposited in the nucleotide database of the National Center for Biotechnology Information (NCBI), most of these being added to support taxonomic or metabarcoding publications $\left(\right.$ eg. $\left.{ }^{51-53}\right)$.

The lengths of the $r b c \mathrm{~L}$ barcodes in Diat.barcode are given in Fig. 1. Most of the sequences are between 1200 and $1600 \mathrm{bp}$ (sequences longer than $1500 \mathrm{bp}$ include other non-coding portions likely the rbcL-rbcS spacer). A few of them are much shorter ( $312 \mathrm{bp}$ ) and correspond to sequences deposited as part of barcoding studies examining the efficiency of shorter fragments for species identification (e.g. $r b c \mathrm{~L} 3 \mathrm{P}^{54,55}$ ), or as part of metabarcoding studies following ${ }^{56}$.

The locations of the sampling sites are given in Fig. 2; they are mostly in Europe and North America, but several are in Asia, including Lake Baikal, Vietnam, Indonesia, Mongolia and Japan (e.g. $\left.{ }^{57-63}\right)$.

Number of sequences per diatom class. To show how barcodes are distributed between major structural types in the diatoms, we used the classification given by ${ }^{64}$ which has three classes, Mediophyceae, Coscinodiscophyceae and Bacillariophyceae, and we also retained the Fragilariophyceae as described ${ }^{65}$; this last class may ${ }^{64}$ or may 


\begin{tabular}{|l|l|l|}
\hline & Marker & $r b c L$ \\
\hline & Imported sequences in v7 (23 feb 2017) & 703 \\
\hline \multirow{5}{*}{ Curation steps } & Sequences in the former version v6 (20 feb 2016) & 2784 \\
\cline { 2 - 3 } & New sequences having a different id. from the sequence of the same clade & 176 \\
\cline { 2 - 3 } & Nomenclatural and taxonomic changes according to peer review papers & 33 \\
\cline { 2 - 3 } & Check of photos - modifications of determinations & 157 \\
\cline { 2 - 3 } & homogenization of taxonomy/synonymies based on phylogenetic results & 1 \\
\hline & Sejected sequences & 5 \\
\hline
\end{tabular}

Table 1. Results of the curation procedure of $23 \mathrm{Feb} 2017$ corresponding to version 7 of the database. Sequences were imported from NCBI (nucleotide database of the National Center for Biotechnology Information) and the TCC (Thonon Culture Collection) between 20 Mar 2016 and 23 Feb 2017. Values in the table give the number of sequences.

\begin{tabular}{|l|l|l|}
\hline & $\boldsymbol{r b c L}$ & $\boldsymbol{r b c L ~ 3 1 2 ~ b p}$ \\
\hline $\begin{array}{l}\text { Total (published in NCBI and in the TCC } \\
\text { and UK barcoding project) }\end{array}$ & 3315 & 167 \\
\hline From TCC and UK barcoding project & 955 & 167 \\
\hline
\end{tabular}

Table 2. Number of sequences in Diat.barcode version 7 for $r b c \mathrm{~L}$ marker.

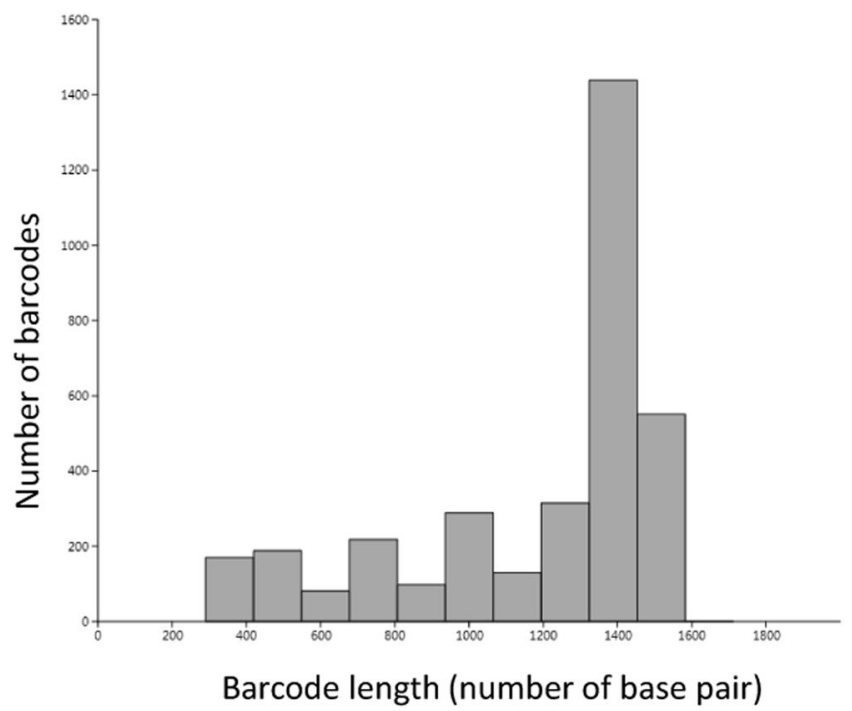

Figure 1. Amount and length of the $r b c \mathrm{~L}$ barcodes present in Diat.barcode version 7 (February 2018).

not be monophyletic but represents a group with similar life-forms and habitat preference (most are attached, non-motile species). We are aware that the taxonomic hierarchy needs considerable modification to make the classes and orders of diatoms monophyletic (e.g. ${ }^{66}$ ), but we nevertheless use traditional groupings to illustrate the coverage of genera and species of different morphological types. The next version of Diat.barcode will have to integrate the new taxonomy of diatoms (and Eukaryotes) given in ${ }^{67}$. Table 3 gives an overview of the number of barcodes and taxa for each class. The Bacillariophyceae is the most barcoded class, with particularly large numbers of barcodes in the orders Bacillariales and Naviculales. Given that the Coscinodiscophyceae comprises a grade of lineages, some quite species-rich, which diverged from each other early in diatom evolution (e.g. ${ }^{66}$ ), this group is arguably the least well barcoded.

For the Bacillariophyceae, many sequences were recently published for Achnanthidium and Planothidium by $^{68}$ and $^{46}$ (this later publication was part of the German Barcode of Life 2 -GBOL2- Diatoms project funded by the German Ministry for Education and Research -BMBF-). Many also come from the UK-barcoding project. Similarly, a number of newly sequenced strains from the genus Nitzschia have been added thanks to studies in marine coastal environments $\left({ }^{69,70}\right.$ and $\left.^{45}\right)$ and from the UK-barcoding project. A major taxonomic study of the Surirellales and Rhopalodiales orders also resulted in many new taxa being sequenced, leading to a major reevaluation of the taxonomy, with creation of new genera and transfers of taxa ${ }^{71}$. Finally, barcodes for 21 taxa that are important for ecological assessment but which have not yet been sequenced (either from cultured strains or from single cells) were obtained directly using HTS data from environmental samples $\left({ }^{56}\right)$, as part of a project funded by the French Biodiversity Agency (Agence Française pour la Biodiversité) in 2017 and 2018. 


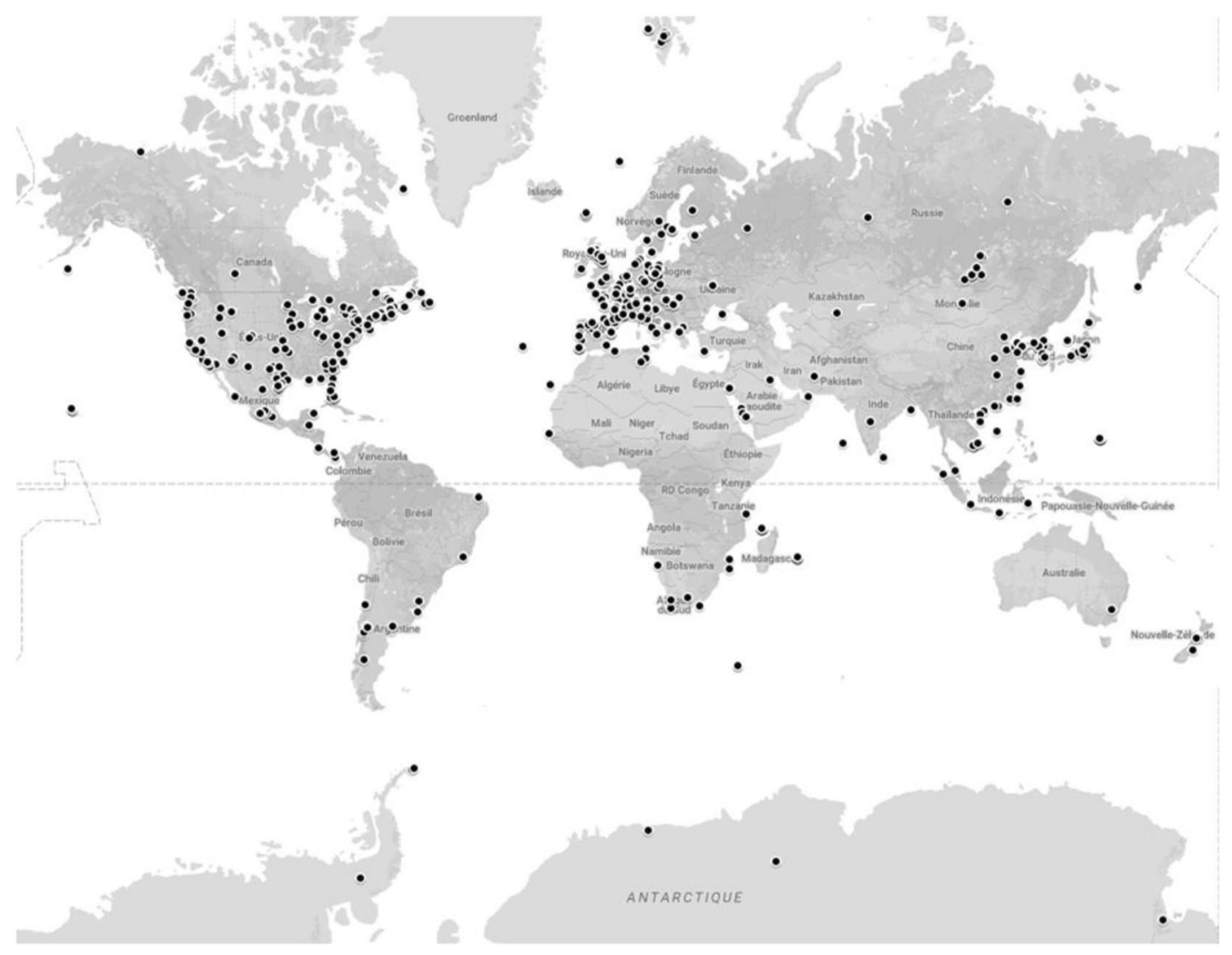

Figure 2. Location of the sampled sites gathered in Diat.barcode (version 7 of February 2018). Map was generated on Google Maps, Map data ( 2019 Google (https://www.google.com/maps), accessible at: https://www.google.com/ maps/d/u/0/edit?mid =1Edfju_8jL_lkwSK5pZzBabECXYhMS3y2\&ll $=-0.7877939911851115 \% 2 C 0 \& z=2$

\section{Conclusions}

A barcode reference library is like a 'molecular dictionary', where each molecular sequence is matched up with its organism. Therefore, a comprehensive and well curated reference database - with high quality sequences and up-to-date, correct taxonomy - is a key factor in both phylogenetic as well as metabarcoding studies (e.g. ${ }^{20,33,36,37,41}$ ). The traceability and availability of metadata (sampling site, isolation protocols, pherograms, vouchers, slides, DNA, photos...) and the accessibility of physical vouchers for the barcodes (culture, raw material, slides, DNA...) are necessary for scientific studies. This point is so important that European diatom experts (FR, DE, HR, HU, UK, ES, CZ, BE...) have worked with the European Standardization Committee to prepare a technical report ${ }^{72}$ as a first step towards standardization of this process. Algal barcoding libraries such as Diat. barcode and Algaterra already fulfill the requirements of this report.

\section{Methods}

Data sources. Data sources used to fill Diat.barcode come from:

- barcoded strains of the Thonon Culture Collection (TCC) ${ }^{73}$; many of these sequences have not yet been published formally in peer-reviewed journals and are not deposited in GenBank;

- barcoded strains of the UK-Barcoding project funded by the UK Environment Agency ${ }^{31}$; similarly, many of these sequences have not yet been published;

- barcoded strains of the Laboratory of Molecular Systematics of Aquatic plants Institute of Plant Physiology Russian Academy of Sciences (curated by Maxim Kulikovsky); many of these sequences have not yet been published formally in peer-reviewed journals;

- diatom sequences published in the nucleotide database of the NCBI.

Barcoded strains and samples of the TCC. The UMR-CARRTEL is a research unit of the French National Institute for Agricultural Research (INRA). It has maintained the TCC since $1968^{73}$. 881 monoclonal strains of freshwater microalgae are registered (486 are alive at the moment of the manuscript submission), among which 543 are diatoms. For each culture, DNA extracts and raw material are kept in the UMR-CARRTEL. Moreover, for diatoms, at least one permanent slide of cleaned frustules (mounted using Naphrax) is kept for each strain, along with nitric acid-treated material (in a vial). This material is accessible for subsequent studies. The strains are 


\begin{tabular}{|c|c|c|c|}
\hline Class & Order & \# of barcodes & \# of taxa \\
\hline \multirow{10}{*}{ Bacillariophyceae } & Achnanthales & 260 & 55 \\
\hline & Bacillariales & 569 & 114 \\
\hline & \begin{tabular}{|l|} 
Cymbellales \\
\end{tabular} & 322 & 90 \\
\hline & Eunotiales & 66 & 12 \\
\hline & Lyrellales & 3 & 3 \\
\hline & Mastogloiales & 1 & 1 \\
\hline & Naviculales & 878 & 263 \\
\hline & \begin{tabular}{|l|} 
Rhopalodiales \\
\end{tabular} & 31 & 11 \\
\hline & Surirellales & 219 & 68 \\
\hline & \begin{tabular}{|l|} 
Thalassiophysales \\
\end{tabular} & 144 & 39 \\
\hline \multirow{9}{*}{ Coscinodiscophyceae } & Asterolamprales & 1 & 1 \\
\hline & Aulacoseirales & 25 & 9 \\
\hline & \begin{tabular}{|l|} 
Chrysanthemodiscales \\
\end{tabular} & 1 & 1 \\
\hline & Corethrales & 2 & 1 \\
\hline & Coscinodiscales & 24 & 14 \\
\hline & Leptocylindrales & 3 & 2 \\
\hline & Melosirales & 28 & 10 \\
\hline & Paraliales & 76 & 6 \\
\hline & Rhizosoleniales & 16 & 9 \\
\hline \multirow{11}{*}{ Fragilariophyceae } & Ardissoneales & 4 & 4 \\
\hline & Climacospheniales & 1 & 1 \\
\hline & Cyclophorales & 5 & 4 \\
\hline & Fragilariales & 333 & 81 \\
\hline & Licmophorales & 10 & 8 \\
\hline & Protoraphidales & 1 & 1 \\
\hline & Rhabdonematales & 3 & 2 \\
\hline & Rhaphoneidales & 8 & 5 \\
\hline & \begin{tabular}{|l|} 
Striatellales \\
\end{tabular} & 13 & 10 \\
\hline & Tabellariales & 11 & 3 \\
\hline & Thalassionematales & 8 & 4 \\
\hline \multirow{9}{*}{ Mediophyceae } & Anaulales & 1 & 1 \\
\hline & Biddulphiales & 14 & 11 \\
\hline & Chaetocerotales & 29 & 15 \\
\hline & \begin{tabular}{|l|} 
Cymatosirales \\
\end{tabular} & 42 & 16 \\
\hline & Hemiaulales & 10 & 9 \\
\hline & Lithodesmiales & 19 & 6 \\
\hline & Thalassiosirales & 212 & 86 \\
\hline & \begin{tabular}{|l|} 
Toxariales \\
\end{tabular} & 2 & 2 \\
\hline & Triceratiales & 83 & 32 \\
\hline
\end{tabular}

Table 3. Number of $r b c \mathrm{~L}$ barcodes and taxa (species or sub species level) per order in Diat.barcode version 7.

available on request through a website dedicated to the collection (http://www6.inra.fr/carrtel-collection_eng/). Each strain is sequenced at least for $r b c \mathrm{~L}$.

In addition to cultures, 66 uncultured samples, which were sequenced using HTS, have been preserved and are kept in the TCC. Indeed, it is possible to relate environmental sequences to the target species observed (and determined) by microscopy and, consequently identify them with high reliability ${ }^{56}$. In such cases, only a short fragment of $r b c \mathrm{~L}$ is sequenced ( $312 \mathrm{bp}$ with Illumina Miseq). This method allows to obtain sequences from diatom species that are difficult to culture. For these uncultured samples, permanent slides and raw material are kept (in ethanol) in the TCC.

All information about these strains and uncultured samples, such as sampling location, isolator, barcode (including type of barcode, amplified region, primer used, protocols, sequencing technology), phenotypic data, photos, associated research programs (for sampling and sequencing) and its taxonomic affiliation are available at https://www6.inra.fr/carrtel-collection_eng/Barcoding-database.

Barcoded strains of the UK-barcoding project funded by the UK environment agency. This project ${ }^{31}$, funded by the UK Environment Agency, England's environmental regulator, aimed to develop a DNA metabarcoding approach to ecological assessment based on diatoms using HTS of a fragment of the $r b c \mathrm{~L}$ gene ${ }^{31}$. It aimed to ensure continuity 
with microscopical methods while, at the same time, complying with the EU Water Framework Directive, which refers to 'taxonomic composition'. The twin foundations for this study were a calibration dataset of samples, analyzed by both microscopy and HTS approaches, along with a reference database of $r b c$ L DNA barcodes which link to Linnaean taxonomy. The samples spanned a wide range of ecological status encountered throughout the UK.

Individual cells of diatoms were isolated by micropipette or by streaking on 2-3\% agar plates. A genus-level identification of living cells was made using an inverted microscope $(\mathrm{x} 40)$. This enabled interesting cells to be selected and transferred to a synthetic medium to grow these cells for subsequent morphological (photos) and molecular (DNA extraction, PCR, Sanger sequencing) analyses. A total of 987 unialgal cultures were obtained from samples collected from 60 locations in England and Scotland. DNA was extracted and sequenced from these cultures. Information about these strains (sampling site location, isolators, barcode, photos) and their taxonomic affiliation are available at https://www6.inra.fr/carrtel-collection_eng/Barcoding-database. DNA has been retained in the Edinburgh DNA (EDNA) bank at the Royal Botanic Garden Edinburgh (E). Voucher slides (mounted in Naphrax) and material are kept in the diatom herbarium at E.

Barcode strains of the laboratory of molecular systematics of aquatic plants of institute of plant physiology russian academy of sciences. A total of 3218 monoclonal strains of freshwater, brackish and marine diatoms from almost all parts of Russia as well as Mongolia, Vietnam, Indonesia, Arctic zone, Spain, Japan, Ethiopia are registered (1678 are alive at the moment of the manuscript submission). For each culture, DNA extracts and raw material are kept in the Institute of Plant Physiology. At least one permanent slide of cleaned frustules (mounted using Naphrax) is kept, along with nitric acid-treated material (in a vial). This material is accessible for subsequent studies. These strains are available on request. Each strain is sequenced at least for $18 \mathrm{~S}$ and $r b c \mathrm{~L}$. All information about these strains, such as sampling location (georeferenced on a google map), isolator, barcode (including type of barcode, amplified region, primer used, protocols, sequencing technology), phenotypic data, photos, associated research programs (for sampling and sequencing) and its taxonomic affiliation are available or will be available for the next version of Diat.barcode (version 9) in 2020 at https://www6.inra.fr/carrtel-collection_eng/Barcoding-database.

Nucleotide database of NCBI. The National Center for Biotechnology Information (NCBI), in the USA, maintains a webserver that collects and provides molecular data and software; these data are publicly accessible via the GenBank database $\left({ }^{74}, \mathrm{http}: / / w w w . n c b i . n l m . n i h . g o v / g e n b a n k\right)$. Initially, all $18 \mathrm{~S}$ (including V4-region of $18 \mathrm{~S}$ ) and $r b c \mathrm{~L}$ nucleotide sequences of diatoms (freshwater and marine) available in GenBank's main collection (CoreNucleotide), whatever their length and their quality, were assessed. We limited the search to these markers because they proved to be effective for species identification (e.g. ${ }^{51,54,75,76}$ ) and showed the best results for metabarcoding ${ }^{12,23,24}$. Sequences for $28 \mathrm{~S}$ rDNA, ITS and cox 1 were not gathered in the database and, since March 2017 (version 5), 18 S sequences are not integrated anymore for practical reasons. Indeed, given the increasing number of diatom sequences available on GenBank and the increasing size of Diat.barcode database, we have decided to work solely on $r b c \mathrm{~L}$ sequences as curating both makers $(18 \mathrm{~S}$ and $r b c \mathrm{~L})$ was too much work and other initiatives are underway to curate $18 \mathrm{~S}^{41}$.

Sequences are retrieved regularly (every 6 months) using the following keywords on the Nucleotide Advanced Search Builder: " $(r b c \mathrm{~L})$ and (diatom OR Bacillariophyta)". Additionally, a publication interval in NCBI is indicated in the Advanced Search Builder: the earliest date corresponds to the previous Diat.barcode update and the most recent to the current date. Diat.barcode is thus updated every 6 months or every year. Unlike $18 \mathrm{~S}$, the number of environmental (cloned) sequences available for $r b c L$ is low compared to the number of sequences derived from cultured strains. Hence we do not search NCBI using BLAST.

Data curation. Sequences that can potentially be integrated into Diat.barcode are from different sources (e.g. GenBank, national barcoding projects, etc...). There are two important drawbacks to consider when gathering new sequences into Diat.barcode and dealing with these is crucial for curation.

First, the earliest data were produced in 1998 and there have been substantial changes in our understanding of diatom taxonomy over the period since then. Moreover, the identifications and taxonomic skills vary between the different authors of the data and have also evolved over time. This means that taxonomy needs to be harmonized before these data are gathered into Diat.barcode.

Second, the quality of the sequences can be suboptimal for correct taxonomic affiliation. Such sequences of low quality are not integrated in Diat.barcode.

These two drawbacks underline the importance of curating the data in order to keep a high quality reference library. The first step of the curation is therefore to check the quality of the sequences. The second step is to curate the species assignment of the strains (from now on "names"): the aim is to achieve, for genetically similar sequences, congruence in their names. The name must be correct according to the most recent taxonomical developments which are themselves subject to expert evaluation. However, as diatom taxonomy is developing rapidly, there will be cases where only a consensus for practical use can be made and solutions regarding the correct name will have to await further studies. In any case, if the original name given by the authors of the sequence was changed during the curation procedure, the traceability of the original name is kept in the database.

This data curation is carried out in two main steps which are very similar to the method used in EukRef ${ }^{41}$ :

- The 1 st step checks the quality and length of sequences and inserts them in a multiple alignment

- The 2nd step, uses a constrained phylogenetic analysis to check that similar sequences (for instance belonging to the same clade) are assigned to the same name. If not, the names are checked through a taxonomic curation procedure.

Figure 3 gives an overview of the general workflow of the curation procedures. Figure 4 gives details on the taxonomic curation procedure listed in Fig. 3. 


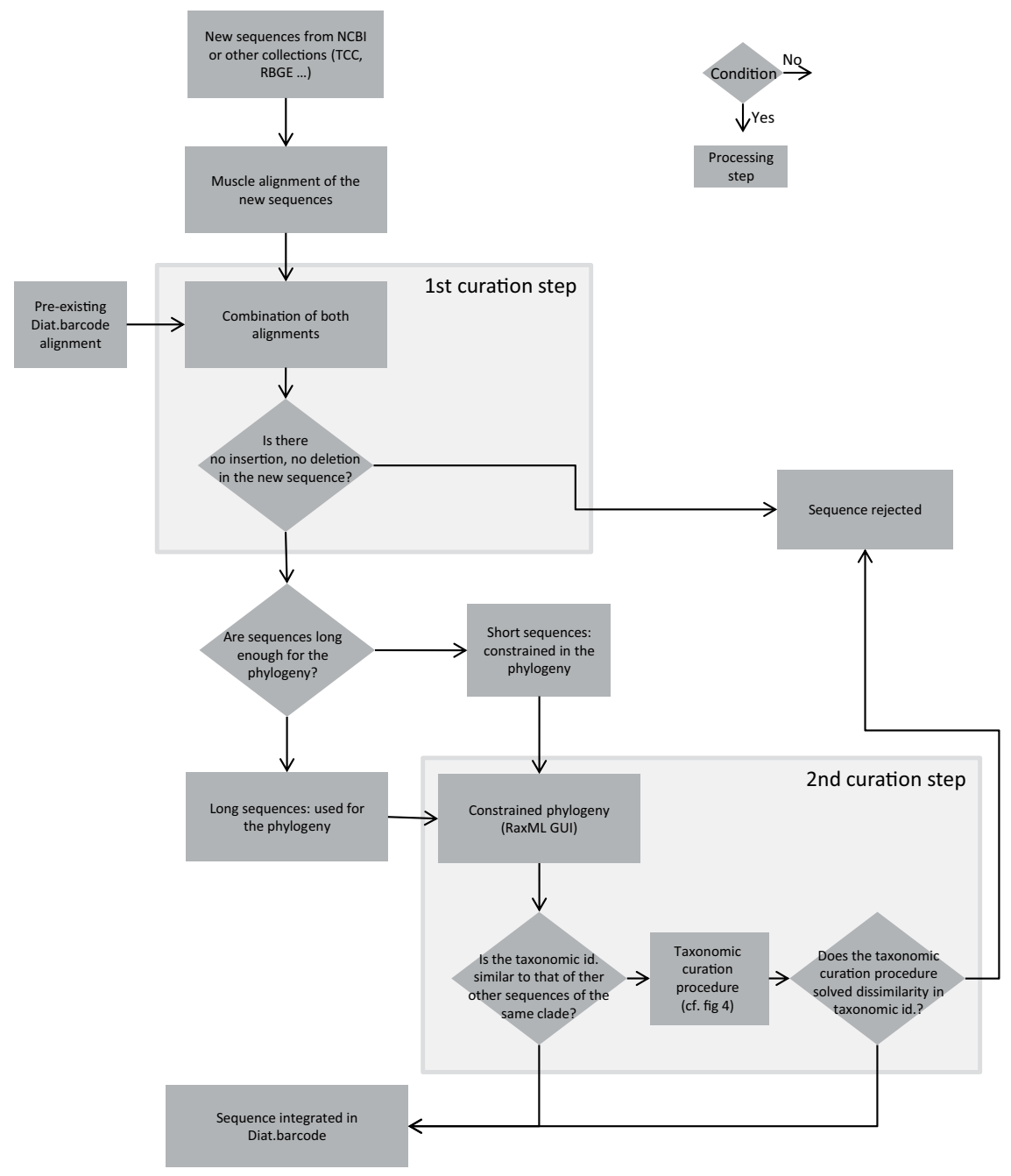

Figure 3. General flowchart of the curation and integration of new sequences in Diat.barcode library. Taxonomic curation procedure is detailed in Fig. 2. Diamonds are conditions, the arrow from the bottom point of the diamond corresponds to "Yes ", the arrow from the right point of the diamond corresponds to "No ". Rectangles are processing steps.

First curation step: checking for sequence quality. A multiple alignment is carried out with the new sequences, usually using Muscle in Seaview ${ }^{77}$. In parallel, a multiple alignment for $r b c \mathrm{~L}$ of the entire Diat.barcode library has been maintained for several years. This alignment has been progressively updated year after year and show no insertions nor deletions since $r b c \mathrm{~L}$ is a coding region, unless insertions or deletion are multiples of 3 nucleotides (until now no frameshifts were encountered so far). The alignment with the new sequences is then added to the alignment of the entire Diat.barcode library. If insertions or deletions appear when adding the new sequences, then the sequences with these insertions or deletions are removed (see box " $1{ }^{\text {st }}$ curation step" in Fig. 3). Sequences with ambiguity codes are removed.

Second curation step: taxonomic curation based on a phylogenetic analysis. Construction of a constrained phylogeny: the objective here is to construct a constrained phylogeny that can be used to assess the taxonomic assignment of the sequences (new and already in Diat.barcode library). We made a constrained phylogeny in order to place the shortest sequences in a phylogeny built with the longest sequences. In the alignment obtained in the first curation step (and which includes new sequences and sequences from Diat.barcode library), sequences are ranked (see diamond "Sequences long enough for the phylogeny" in Fig. 3) into:

- long sequences, starting before nucleotide position 250 of the alignment and ending after nucleotide position 1129; these sequences are used to construct a phylogeny including 879 alignment positions;

- short sequences, starting after nucleotide position 250 and/or ending before nucleotide position 1129; these sequences are not used for the phylogeny construction and will be placed afterwards, using a phylogeny constrained by the topology of the tree inferred only with long sequences. 


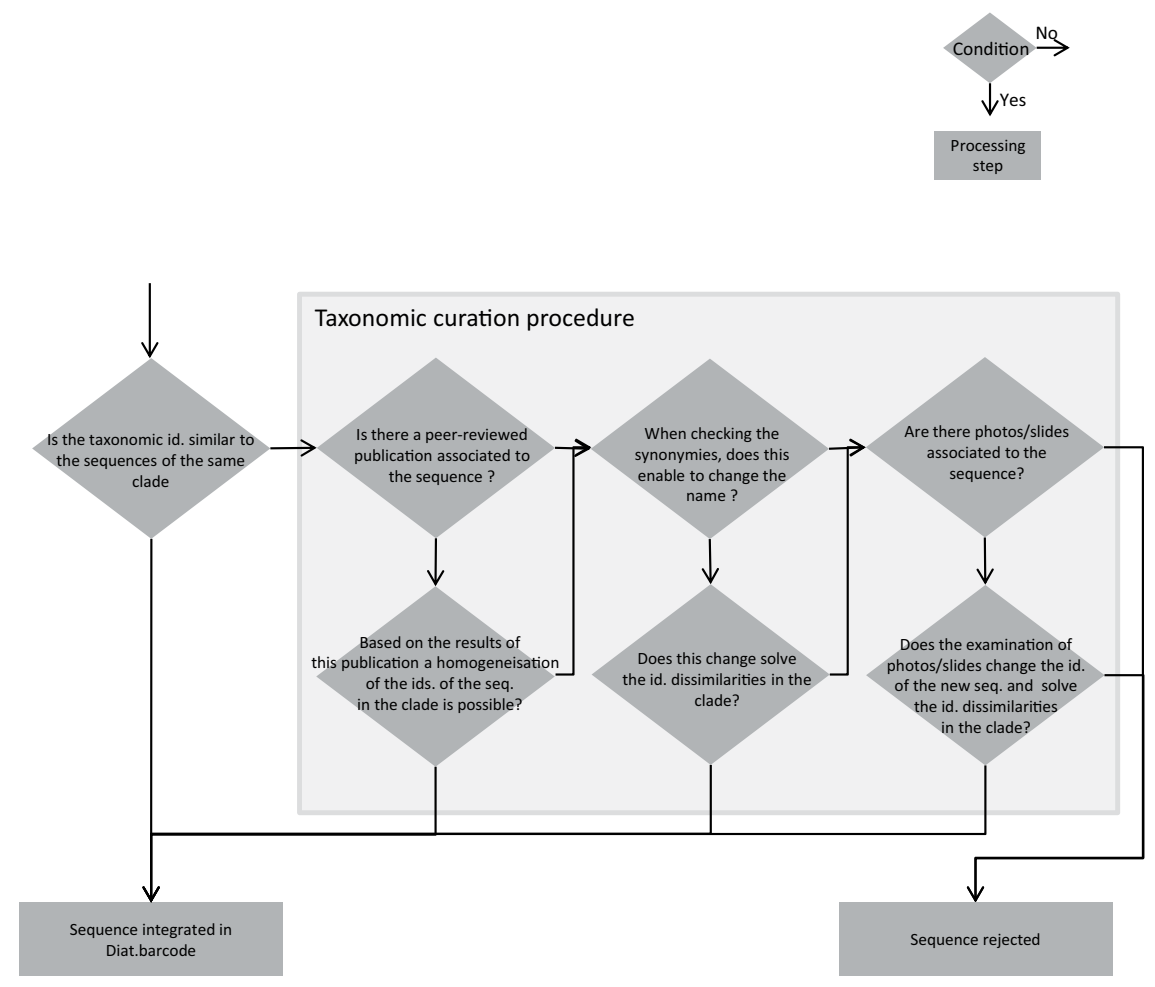

Figure 4. Flowchart of the taxonomic curation procedure. Diamonds are conditions, the arrow from the bottom point of the diamond corresponds to « Yes", the arrow from the right point of the diamond corresponds to « No ». Rectangles are processing steps.

The short sequences are marked with an "*” at the beginning of their sequence name (it is important to identify such sequences to ensure that they are not used to construct the phylogeny). The constrained phylogeny is made using raxmlGUI ${ }^{78}$. In the raxmlGUI menu, the option "Enforce constraint" is selected, followed by "Define topological constraint", and finally the short sequences are used as "constrained taxa". Then a ML + rapid bootstrap with $100+$ GTR Gamma model is launched (such calculation takes 3 to 4 days' calculation using 2 threads on a computer equipped with 8 Intel ${ }^{\circledR} \mathrm{Xeon}^{\circledR}$ CPU E5-1620 3.60 ghz).

Taxonomic curation: The taxonomic curation procedure (Fig. 4) focuses only on the clades bearing new sequences (long or short sequences). The new sequences are marked with an “@” at the beginning of their sequence name in order to recognize them easily in the phylogeny. New short sequences are therefore prefixed “*@”.

Here the overall objective is to check that for each well-supported monophyletic clade (bootstrap values above $50 \%$ ) phylogenetic neighbor sequences of the new sequences «@» have homogeneous names. If they are not homogeneous, several verifications are done as explained below:

i. Check if a peer-reviewed publication is associated with the new sequence. If this is the case, the results of the publication can be used to assess if the names of the new and/or old sequences can be homogenized for the clade containing them. Depending on the case, homogenization might be achieved at the species level or, if this is not possible, at the generic level or even higher level. This homogenization can take place in any of several points ( $\mathrm{i}$, ii of iii). If these modifications result in a clade with homogenized names, the new sequence(s) is (are) kept.

ii. If no such peer-reviewed publications are available, the synonymies of the names of the sequences inside the clade are checked. We use of the online taxonomic catalogue DiatomBase at diatombase.org ${ }^{79}$ which integrates the former Catalogue of Diatom Names ${ }^{80}$. The online taxonomic catalogue AlgaeBase can be used as well ${ }^{81}$ only if DiatomBase lacks the information looked for. If this results in homogenized taxonomy, the sequence and the new taxonomic names are kept.

iii. If this is not the case, we check if photos/slides are associated with the sequence(s). There are several websites from which photos of strains are available, for instance:

- AlgaTerra accessible at http://www.algaterra.org is maintained by the Botanic Garden and Botanical Museum Berlin-Dahlem (Germany, W.-H. Kusber, R. Jahn, N. Abarca, O. Skibbe \& J. Zimmermann);

- Protist central accessible at http://www.protistcentral.org/ is maintained by the Texas Natural Science Center of the University of Texas (USA, E. Theriot); 
- the website of Papanin Institute for Biology of Inland Waters Russian Academy of Sciences http://ibiw.ru/ index.php?p = project/algo/WDCM602/mgmt_class\&id = 7\&lang = en (Russia, M Kulikovsky);

- Bold accessible at http://www.boldsystems.org/ ${ }^{82}$ is maintained by the University of Guelph.

If the re-examination of the photos/slides results in a change in the name associated with the sequence that better fits its position in the $r b c \mathrm{~L}$-based phylogenetic tree, the sequence and its new taxonomic name is kept. If the taxonomic name is still different after checking photos/slide, the sequence is rejected.

Each time a modification of the name is made, a record of all changes is kept. The original name given by the author of the sequence is kept in Diat.barcode files.

Data access. The websites giving access to download the data are accessible at https://www6.inra.fr/ carrtel-collection_eng/Barcoding-database.

For each strain, the following information is given, if available: sampling site information (name and coordinates), type of habitat, strain code given by the laboratory, name of the project which funded the field sampling, the laboratory responsible for field sampling, DNA extraction, PCR, sequencing, and the dates of the different steps. A species name is given to each strain, except in a few cases where only the genus is given. In addition, the taxonomic affiliation is given up to the kingdom, following the hierarchical system given in Algaebase ${ }^{81}$. For molecular criteria, the database gives the type of marker (18S, 28S, ITS2, cox1, $r b c \mathrm{~L}$ ), the primers used for sequencing and PCR. Protocols for DNA extraction and PCR are also given and the laboratory responsible for the sequence is named. Phenotypic information is given (including average species dimensions, chloroplast shapes and number, life-form, ecological class, sensitivity values for widely-used biotic indices). Photos (living material or/and cleaned frustules or valves) of the strains coming from the TCC ${ }^{73}$, the UK-Barcoding project funded by the UK Environment Agency ${ }^{31}$ and from Maxim Kulikovsky, are also downloadable and the web links are given at https://www6.inra.fr/carrtel-collection_eng/Barcoding-database.

A ready-to-use database for metabarcoding: Rsyst::diatom_rbcl_align_312bp. As stated earlier, the use of HTS methods and metabarcoding is getting more and more common to study the composition of environmental samples. Current HTS platforms such as Illumina MiSeq enable fragments of around $300 \mathrm{bp}$ to be sequenced. The most common barcode used today for diatoms is a $312 \mathrm{bp}$ long stretch of the $r b c \mathrm{~L}$ marker (e.g. ${ }^{13,25,27,31,38,56}$ ). Therefore, the ability to extract this particular region from Diat.barcode is necessary. The authors of this paper, in particular V. Vasselon, have made this extraction from the multiple alignment of the different sequences present in Diat.barcode. Some species, which can be distinguished on the basis of full length of $r b c \mathrm{~L}(\sim 1500 \mathrm{bp})$ sequences, can have identical sequences when this $312 \mathrm{bp}$ barcode is used. This means that a further curation procedure is required. This procedure, called "Rsyst::diatom_rbcl_align_312bp", is described below:

First, the $312 \mathrm{bp} r b c \mathrm{~L}$ barcode is extracted from the full Diat.barcode database $r b c \mathrm{~L}$ alignment (by reference to the positions of the Diat_rbcL_708 $\mathrm{F}^{83}$ and R $3^{84}$ primers). Sequences with ambiguities (N), homopolymers longer than 8 and sequences shorter than $312 \mathrm{bp}$ are removed from the database. The resulting sequences are dereplicated into Individual Sequence Units (ISUs) in order to identify taxa sharing identical DNA sequences in the $312 \mathrm{bp} r b c \mathrm{~L}$ barcode region. If necessary, we harmonize the taxonomy between all taxa found in each single ISU. Finally, only ISUs are retained in the database, each represented by one taxon ID and one DNA sequence. The resulting ISUs database is assigned to itself using the Mothur assignment algorithm (classify.seqs command). The new taxonomy is compared with the expected assignments to evaluate potential sources of bias (erroneous taxonomic names, taxa impossible to differentiate,...). If necessary, ambiguous sequences are removed from the database or the taxonomy is adjusted. Finally, we harmonize potentially conflicting names (e.g. "aff." and "cf." are removed; "Nanofrustulum_sp._SZCZCH285" transformed into "Nanofrustulum_sp.").

The resulting files are a ".fasta" file which contains the $r b c \mathrm{~L} 312 \mathrm{bp}$ DNA sequences and a ".txt" file which contains the corresponding taxonomy and the sequence identifier common to both files. The sequence identifier is composed of an accession number (also present in the Diat.barcode library) and the original name given by the author of the sequence (eg: TCC679-Rbcl-1|Achnanthidium_pyrenaicum). The text file gathers the curated taxonomical information from empire to species level.

For instance, for TCC679-Rbcl-1|Achnanthidium_pyrenaicum the taxonomy is Eukaryota; Chromista; Chromobiota; Bacillariophyta; Bacillariophyceae; Achnanthales; Achnanthidiaceae; Achnanthidium; Achnant hidium_minutissimum. In this case, Achnanthidium_pyrenaicum is the original species name given by the author and Achnanthidium_minutissimum is the curated species name that will be used in metabarcoding. This database has been curated for a specific use with filtering procedures to meet our own needs (especially for use in metabarcoding for diatom based ecological assessment in rivers and lakes) and is provided on this basis. The original database, Diat.barcode, is the reference and can be curated differently to meet different requirements.

Received: 5 March 2019; Accepted: 25 September 2019;

Published online: 22 October 2019

\section{References}

1. Mann, D. G. \& Vanormelingen, P. An inordinate fondness? The number, distributions and origins of diatom species. J. Eukaryot. Microbiol. 60, 1-26(2013).

2. Stevenson, R. J. Ecological assessments with algae: a review and synthesis. J. Phycol. 50, 437-461 (2014).

3. Kolkwitz, R. \& Marson, M. Ökologie der pflanzliche Saprobien. Berichte der Deutsche Botanische Gesellschaften 26, 505-519 (1908).

4. Butcher, R. W. Studies in the ecology of rivers. IV. The algae of organically enriched water. J. Ecol. 35, 186-191 (1947).

5. Hustedt, F. Die Diatomeenflora des Flusssystems der Weser im Gebiet der Hansestadt Bremen. Abhandlungen naturwissenschaftlichen Verein zu Bremen 34, 181-440 (1957). 
6. Zelinka, M. \& Marvan, P. Zur Prazisierung der biologischen Klassifikation der Reinheit fliessender Gewasser. Arch. Hydrobiol. 57, 389-407 (1961).

7. Rimet, F. Recent views on river pollution and diatoms. Hydrobiologia 683, 1-24 (2012).

8. European commission. Directive 2000/60/EC of the European Parliament and of the Council of 23rd October 2000 establishing a framework for Community action in the field of water policy. Official Journal of the European Communities 327, 1-72 (2000).

9. Barbour, M. T., Gerritsen, J., Snyder, B. D. \& Stribling, J. B. Rapid bioassessment protocols for use in streams and wadeable rivers: periphyton, benthic macroinvertebrates and fish. Second edition. 1 (US Environmental Protection Agency, Office of Water, Washington, DC, 1999).

10. Leblanc, K. et al. A global diatom database - abundance, biovolume and biomass in the world ocean. Earth Syst. Sci. Data 4, 149-165 (2012).

11. Afnor. EN 14407 - Water quality Guidance standard for the identification, enumeration and interpretation of benthic diatom samples from running waters. CEN standard 1-13 (2014).

12. Kermarrec, L. et al. Next-generation sequencing to inventory taxonomic diversity in eukaryotic communities: a test for freshwater diatoms. Mol. Ecol. Res. 13, 607-619 (2013).

13. Pawlowski, J. et al. The future of biotic indices in the ecogenomic era: Integrating (e)DNA metabarcoding in biological assessment of aquatic ecosystems. Sci. Total Environ. 637-638, 1295-1310 (2018)

14. Besse-Lotoskaya, A., Verdonschot, P. \& Sinkeldam, J. Uncertainty in diatom assessment: sampling, identification and counting variation. Hydrobiologia 566, 247-260 (2006).

15. Kahlert, M. et al. Harmonization is more important than experience-results of the first Nordic-Baltic diatom intercalibration exercise 2007 (stream monitoring). J. Appl. Phycol. 21, 471-482 (2009).

16. Hebert, P., Cywinska, A., Ball, S. L. \& deWaard, J. R. Biological identifications through DNA barcodes. P. Roy. S. B-Biol. Sci. 270, 313-321 (2003).

17. Kress, W. J., Wurdack, K. J., Zimmer, E. A., Weigt, L. A. \& Janzen, D. H. Use of DNA barcodes to identify flowering plants. Proc Natl Acad Sci USA 102, 8369-8374 (2005).

18. Evans, K. M. \& Mann, D. G. A proposed protocol for nomenclaturally effective DNA barcoding of microalgae. Phycologia 48, 70-74 (2009).

19. Moniz, M. B. J. \& Kaczmarska, I. Barcoding diatoms: Is there a good marker? Mol. Ecol. Res. 9, 65-74 (2009).

20. Zimmermann, J. et al. Taxonomic reference libraries for environmental barcoding: a best practice example from diatom research. PLoS ONE 9, 1-24 (2014).

21. Vasselon, V. et al. Avoiding quantification bias in metabarcoding: Application of a cell biovolume correction factor in diatom molecular biomonitoring. Methods Ecol. Evol. 9, 1060-1069 (2018).

22. Pompanon, F., Coissac, E. \& Taberlet, P. Metabarcoding a new way to analyze biodiversity. Biofutur 30-32 (2011)

23. Kermarrec, L. et al. A next-generation sequencing approach to river biomonitoring using benthic diatoms. Freshwater Sci. 33, 349-363 (2014).

24. Zimmermann, J., Glöckner, G., Jahn, R., Enke, N. \& Gemeinholzer, B. Metabarcoding vs. morphological identification to assess diatom diversity in environmental studies. Mol. Ecol. Res. 15, 526-542 (2014).

25. Rivera, S. et al. Metabarcoding of lake benthic diatoms: from structure assemblages to ecological assessment. Hydrobiologia 807, 37-51 (2018)

26. Rimet, F., Vasselon, V., A.-Keszte, B. \& Bouchez, A. Do we similarly assess diversity with microscopy and high-throughput sequencing? Case of microalgae in lakes. Org. Divers. Evol. 18, 51-62 (2018).

27. Rivera, S. F. et al. DNA metabarcoding and microscopic analyses of sea turtles biofilms: Complementary to understand turtle behavior. PLOS ONE 13, e0195770 (2018).

28. Endo, H., Ogata, H. \& Suzuki, K. Contrasting biogeography and diversity patterns between diatoms and haptophytes in the central Pacific Ocean. Sci. Rep. 8, 10916 (2018).

29. Capo, E. et al. Tracking a century of changes in microbial eukaryotic diversity in lakes driven by nutrient enrichment and climate warming. Environ. Microbiol. 19, 2873-2892 (2017).

30. Dulias, K., Stoof-Leichsenring, K. R., Pestryakova, L. A. \& Herzschuh, U. Sedimentary DNA versus morphology in the analysis of diatom-environment relationships. J Paleolimnol. 57, 51-66 (2017).

31. Kelly, M. G. et al. A DNA based diatom metabarcoding approach for Water Framework Directive classification of rivers. SC140024/R, Environment Agency, Bristol. ISBN: 978-1-84911-406-6. 94 pp (2016).

32. Quast, C. et al. The SILVA ribosomal RNA gene database project: improved data processing and web-based tools. Nucleic Acids Res. 41, D590-D596 (2013).

33. Guillou, L. et al. The Protist Ribosomal Reference database (PR2): a catalog of unicellular eukaryote Small Sub-Unit rRNA sequences with curated taxonomy. Nucleic Acids Res. 41, D597-D604 (2013).

34. Decelle, J. et al. PhytoREF: a reference database of the plastidial 16S rRNA gene of photosynthetic eukaryotes with curated taxonomy. Mol. Ecol. Res. 15, 1435-1445 (2015).

35. Morard, R. et al. PFR2: a curated database of planktonic foraminifera $18 \mathrm{~S}$ ribosomal DNA as a resource for studies of plankton ecology, biogeography and evolution. Mol. Ecol. Res. 15, 1472-1485 (2015).

36. Boscaro, V. et al. EukRef-Ciliophora: A manually curated, phylogeny-based database of small subunit rRNA gene sequences of ciliates. Env. Microbiol. 20, 2218-2230 (2018).

37. Mordret, S. et al. dinoref: A curated dinoflagellate (Dinophyceae) reference database for the $18 \mathrm{~S}$ rRNA gene. Mol. Ecol. Res. 18, 974-987 (2018)

38. Vasselon, V., Rimet, F., Tapolczai, K. \& Bouchez, A. Assessing ecological status with diatoms DNA metabarcoding: Scaling-up on a WFD monitoring network (Mayotte island, France). Ecol. Indic. 82, 1-12 (2017).

39. Vasselon, V., Domaizon, I., Rimet, F., Kahlert, M. \& Bouchez, A. Application of high-throughput sequencing (HTS) metabarcoding to diatom biomonitoring: Do DNA extraction methods matter? Freshwater Sci. 36, 162-177 (2017).

40. Rimet, F. et al. R-Syst::diatom: An open-access and curated barcode database for diatoms and freshwater monitoring. DatabaseOxford. 2016, 21 (2016).

41. del Campo, Jdel et al. EukRef: Phylogenetic curation of ribosomal RNA to enhance understanding of eukaryotic diversity and distribution. PLOS Biology 16, e2005849 (2018).

42. Trobajo, R. et al. The use of partial cox $1, \mathrm{rbcL}$ and LSU rDNA sequences for phylogenetics and species identification within the Nitzschia palea species complex (Bacillariophyceae). Eur. J. Phycol. 45, 413-425 (2010).

43. Trobajo, R. et al. Morphology and identity of some ecologically important small Nitzschia species. Diatom Res. 28, 37-59 (2013).

44. Rovira, L., Trobajo, R., Sato, S., Ibanez, C. \& Mann, D. G. Genetic and Physiological Diversity in the Diatom Nitzschia inconspicua. J. Eukaryot. Microbiol. 62, 815-832 (2015).

45. Carballeira, R. et al. A combined morphological and molecular approach to Nitzschia varelae sp. nov., with discussion of symmetry in Bacillariaceae. Eur. J. Phycol. 52, 342-359 (2017).

46. Jahn, R. et al. Planothidium lanceolatum and Planothidium frequentissimum reinvestigated with molecular methods and morphology: four new species and the taxonomic importance of the sinus and cavum. Diatom Res. 32, 75-107 (2017).

47. Pfannkuchen, D. M. et al. The Ecology of One Cosmopolitan, One Newly Introduced and One Occasionally Advected Species from the Genus Skeletonema in a Highly Structured Ecosystem, the Northern Adriatic. Microb. Ecol. 75, 674-687 (2018). 
48. Grbin, D. et al. Multigene phylogeny and morphology of newly isolated strain of Pseudo-nitzschia mannii Amato \& Montresor (Adriatic Sea). Diatom Res. 32, 127-131 (2017).

49. Ivancic, I. et al. Alkaline phosphatase activity related to phosphorus stress of microphytoplankton in different trophic conditions. Prog. Oceanogr. 146, 175-186 (2016).

50. Kahlert, M. et al. Connecting the morphological and molecular species concepts to facilitate species identification within the genus Fragilaria (Bacillariophyta). J. Phycol. 55, 948-970 (2019)

51. Kermarrec, L., Bouchez, A., Rimet, F. \& Humbert, J. F. First Evidence of the Existence of Semi-Cryptic Species and of a Phylogeographic Structure in the Gomphonema parvulum (Kutzing) Kutzing Complex (Bacillariophyta). Protist 164, 686-705 (2012).

52. Larras, F., Keck, F., Montuelle, B., Rimet, F. \& Bouchez, A. Linking Diatom Sensitivity to Herbicides to Phylogeny: A Step Forward for Biomonitoring? Envir. Sci. Tech. 48, 1921-1930 (2014).

53. Rimet, F. et al. When is Sampling Complete? The Effects of Geographical Range and Marker Choice on Perceived Diversity in Nitzschia palea (Bacillariophyta). Protist 165, 245-259 (2014).

54. Hamsher, S. E., Evans, K. M., Mann, D. G., Poulickova, A. \& Saunders, G. W. Barcoding Diatoms: Exploring Alternatives to COI-5P. Protist 162(3), 405-422 (2011).

55. MacGillivary, M. L. \& Kaczmarska, I. Survey of the Efficacy of a Short Fragment of the rbcL Gene as a Supplemental DNA Barcode for Diatoms. J. Eukaryot. Microbiol. 58, 529-536 (2011).

56. Rimet, F. et al. The potential of high throughput sequencing (HTS) of natural samples as a source of primary taxonomic information for reference libraries of diatom barcodes. Fottea. https://doi.org/10.5507/fot.2017.013 (2018).

57. Andreeva, S. et al. Sellaphora balashovae (Bacillariophyta), a new species from Siberian mountain Lake Frolikha (Baikal region), Russia. Phytotaxa 371, 73-83 (2018).

58. Kulikovskiy, M., Glushchenko, A. M., Kuznetsova, I. \& Kociolek, J. P. New Freshwater Diatom Genus Okhapkinia Gen. Nov from Laos (southeast Asia), with Notes on Molecular Phylogeny of the Family Sellaphoraceae. Phycologia 56, 109-109 (2017).

59. Kulikovskiy, M., Andreeva, S., Gusev, E., Kuznetsova, I. V. \& Annenkova, N. V. Molecular Phylogeny of Monoraphid Diatoms and Raphe Significance in Evolution and Taxonomy. Biology Bull. 43, 398-407 (2016).

60. Kulikovskiy, M., Lange-Bertalot, H., Annenkova, N. V., Gusev, E. \& Kociolek, J. P. Morphological and molecular evidence support description of two new diatom species from the genus Ulnaria in Lake Baikal. Fottea 1, 34-42 (2016).

61. Kulikovskiy, M., Gusev, E., Andreeva, S. \& Annenkova, N. Phylogenetic position of the diatom genus Geissleria Lange-Bertalot \& Metzeltin and description of two new species from Siberian mountain lakes. Phytotaxa 177, 249-260 (2015).

62. Maltsev, Y., Svetlana, A., Kulikovskiy, M., Podunai, J. \& Kociolek, J. P. Molecular phylogeny of the diatom genus Envekadea (Bacillariophyceae, Naviculales). Nova Hedwigia 46, 241-252 (2017).

63. Petrushkina, M. et al. Fucoxanthin production by heterokont microalgae. Algal Res. 24, 387-393 (2017).

64. Medlin, L. K. A Review of the Evolution of the Diatoms from the Origin of the Lineage to Their Populations. In The Diatom World (eds Seckbach, J. \& Kociolek, J. P.) 93-118 (Springer Science + Business Media B.V. 2011).

65. Round, F., Crawford, C. G. \& Mann, D. G. The diatoms. Biology and morphology of the genera. (Cambridge University Press, 1990).

66. Theriot, E. C., Ashworth, M. P., Nakov, T., Ruck, E. \& Jansen, R. K. Dissecting signal and noise in diatom chloroplast protein encoding genes with phylogenetic information profiling. Mol. Phylogenet. Evol. 89, 28-36 (2015).

67. Adl, S. M. et al. Revisions to the Classification, Nomenclature, and Diversity of Eukaryotes. J. Eukaryot. Microbiol. 66, 4-119 (2019).

68. Pinseel, E. et al. Molecular and morphological characterization of the Achnanthidium minutissimum complex (Bacillariophyta) in Petuniabukta (Spitsbergen, High Arctic) including the description of A. digitatum sp. nov. Eur. J. Phycol. 1-17 (2017).

69. Witkowski, A. et al. Multigene Assessment of Biodiversity of Diatom(Bacillariophyceae) Assemblages from the Littoral Zone of the Bohai and Yellow Seas in Yantai Region of Northeast China with some Remarks on Ubiquitous Taxa. J. Coast. Res. 74, 166-195 (2016).

70. An, S. M., Choi, D. H., Lee, J. H., Lee, H. \& Noh, J. H. Identification of benthic diatoms isolated from the eastern tidal flats of the Yellow Sea: Comparison between morphological and molecular approaches. PLOS ONE 12, e0179422 (2017).

71. Ruck, E., Nakov, T., Alverson, A. J. \& Theriot, E. C. Phylogeny, ecology, morphological evolution, and reclassification of the diatom orders Surirellales and Rhopalodiales. Mol. Phylogenet. Evol. 103, 155-171 (2016).

72. CEN. Water quality - CEN/TR 17244 - Technical report for the management of diatom barcodes. 1-11 (2018).

73. Rimet, F. et al. Thonon Culture Collection -TCC- a freshwater microalgae collection, https://doi.org/10.15454/UQEMVW, Portail Data Inra, V1 (2018).

74. Sayers, E. W. et al. Database resources of the National Center for Biotechnology Information. Nucleic Acids Res. 47, D23-D28 (2019).

75. Evans, K. M., Wortley, A. H. \& Mann, D. G. An assessment of potential diatom 'barcode' genes (cox1, rbcL, 18S and ITS rDNA) and their effectiveness in determining relationships in Sellaphora (Bacillariophyta). Protist 158, 349-364 (2007).

76. Zimmermann, J., Jahn, R. \& Gemeinholzer, B. Barcoding diatoms: evaluation of the V4 subregion on the $18 \mathrm{~S}$ rRNA gene, including new primers and protocols. Org. Divers. Evol. 11, 1-20 (2011).

77. Gouy, M., Guindon, S. \& Gascuel, O. SeaView Version 4: A Multiplatform Graphical User Interface for Sequence Alignment and Phylogenetic Tree Building. Mol. Biol. Evol. 27, 221-224 (2010).

78. Silvestro, D. \& Michalak, I. raxmlGUI: a graphical front-end for RAxML. Org. Divers. Evol. 12, 335-337 (2012).

79. Kociolek, J. P. et al. DiatomBase. website: www.diatombase.org. (2018).

80. Kociolek, J. P., Sabbe, K., Vandepitte, L., Decock, W. \& Vanhoorn, B. Catalogue of Diatom Names Resurrected: DiatomBase will be the new authority resource for diatom names and more. $24 \mathrm{~h}$ International Diatom Symposium 100 (2016).

81. Guiry, M. D. \& Guiry, G. M. AlgaeBase. World-wide electronic publication, National University of Ireland, Galway, http://www. algaebase.org; searched on 24 november 2014. (2014).

82. Ratnasingham, S. \& Hebert, P. D. N. BOLD: The Barcode of Life Data System (www.barcodinglife.org). Mol. Ecol. Notes 7, 355-364 (2007).

83. Stoof-Leichsenring, K. R., Epp, L. S., Trauth, M. H. \& Tiedemann, R. Hidden diversity in diatoms of Kenyan Lake Naivasha: a genetic approach detects temporal variation. Mol. Ecol. 21, 1918-1930 (2012).

84. Bruder, K. \& Medlin, L. K. Molecular assessment of phylogenetic relationships in selected species/genera in the naviculoid diatoms (Bacillariophyta). I. The genus Placoneis. Nova Hedwigia 85, 331-352 (2007).

85. Lefrançois, E. et al. Development and implementation of eco-genomic tools for aquatic ecosystem biomonitoring: the SYNAQUA French-Swiss program. Environ. Sci. Pollut. R. 25, 33858-33866 (2018).

\section{Acknowledgements}

The following research programs supported isolation and sequencing of the diatom strains: @speedID, Bibliothèque du vivant (French Barcoding of Life projects), IperRetro (ANR project, France), Mayotte project (ONEMA France), Diamed, Modelecotox (FNR projects, Luxembourg), L. Kermarrec PhD grant (Asconit Consultants and ANRT, France), UK diatom barcoding project of the UK Environment Agency (project SC140024/R). The authors thank Cécile Chardon, Rabia Demir, Agnès Rimet, Sonia Lacroix, Isabelle Huguet for their implication in the update of the database. Maria Kahlert received support from the SYNTHESYS Project 
http://www.synthesys.info/ which is financed by European Community Research Infrastructure Action under the FP7 "Capacities" Program and from The Swedish Agency for Marine and Water Management and SLU's Environmental monitoring and assessment (EMA) program "Lakes and watercourses". Work with diatom collection and curation under Maxim Kulikovskiy was supported by RFBR (19-34-70016-mol_a_mos for morphological analysis) and RSF (19-14-00320 for molecular investigation). The curation of the monoraphid diatoms by Jonas Zimmermann was supported by the Federal Ministry of Education and Research (German Barcode of Life 2 Diatoms (GBOL2), grant number 01LI1501E, website: www.bolgermany.de). This article is based upon work from COST Action DNAqua-Net (CA15219), supported by the COST (European Cooperation in Science and Technology) program, from the French-Swiss program SYNAQUA ${ }^{85}$ (INTERREG FranceSwitzerland 2017-2019) and from the Alpine-Space program (INTERREG Alpine-Space 2018-2021).

\section{Author contributions}

All authors (F. Rimet, E. Gusev, M. Kahlert, M.G. Kelly, M. Kulikovskiy, Y. Maltsev, D.G. Mann, M. Pfannkuchen, R. Trobajo, V. Vasselon, J. Zimmermann, A. Bouchez) participated in manuscript writing and database curation.

\section{Competing interests}

The authors declare no competing interests.

\section{Additional information}

Correspondence and requests for materials should be addressed to F.R.

Reprints and permissions information is available at www.nature.com/reprints.

Publisher's note Springer Nature remains neutral with regard to jurisdictional claims in published maps and institutional affiliations.

(c) (i) Open Access This article is licensed under a Creative Commons Attribution 4.0 International License, which permits use, sharing, adaptation, distribution and reproduction in any medium or format, as long as you give appropriate credit to the original author(s) and the source, provide a link to the Creative Commons license, and indicate if changes were made. The images or other third party material in this article are included in the article's Creative Commons license, unless indicated otherwise in a credit line to the material. If material is not included in the article's Creative Commons license and your intended use is not permitted by statutory regulation or exceeds the permitted use, you will need to obtain permission directly from the copyright holder. To view a copy of this license, visit http://creativecommons.org/licenses/by/4.0/.

(c) The Author(s) 2019 